\title{
FÁBRICAS DE ARTEFATOS DE CIMENTO DO MUNICÍPIO DE CRUZ DAS ALMAS: CARACTERIZAÇÃO E SUGESTÕES PARA MELHORIAS
}

\author{
Danilo Machado Cavalcante ${ }^{1}$ \\ Gabriel Pereira da Conceição ${ }^{2}$ \\ Álvaro Gabriel Coutinho dos Santos ${ }^{3}$ \\ Fernanda Nepomuceno Costa ${ }^{4}$
}

Resumo: Os artefatos de cimento são termos preliminarmente empregados aos mais diversos produtos fabricados, como tubos de concreto, blocos, telhas, pisos, postes, estacas, ladrilhos, tijolos entre outros. $A$ indústria de artefatos de cimento em Cruz das Almas é constituída por micro e pequenas empresas e as principais carências desse seguimento se dão com relação à qualidade de seus produtos, o domínio na fabricação, o desperdício dos materiais e a gestão dos resíduos. Este trabalho tem como objetivo caracterizar as fábricas da região e proporcionar melhores práticas na produção de artefatos de cimento. Para isso utilizou-se do método de investigação proposto por Costa (2015), e com o auxílio da normalização técnica do país foi possível efetuar o diagnóstico necessário para a realização do mesmo. Outro aspecto importante analisado neste trabalho se deu com relação à segurança dos trabalhadores nos estabelecimentos examinados. Com o diagnóstico efetuado, buscou-se soluções para as fábricas analisadas, nas áreas detectadas com carências.

Palavras-chave: Artefatos de cimento; Fábricas; Diagnóstico; Caracterização.

\footnotetext{
1 Engenharia Civil / Universidade Federal do Recôncavo da Bahia - UFRB, Brasil. E-mail: cavalcante21machado@hotmail.com.

2 Engenharia Civil / Universidade Federal do Recôncavo da Bahia Bahia - UFRB, Brasil. E-mail: gabrielpereira72@hotmail.com.

3 Engenharia Civil / Universidade Federal do Recôncavo da Bahia Bahia - UFRB, Brasil. E-mail: alvaro_ufrb@hotmail.com.

${ }^{4}$ Universidade Federal do Recôncavo da Bahia - UFRB, Brasil. E-mail: fernandacosta@ufrb.edu.br.
} 